\title{
La perspectiva de estudiantes y jóvenes investigadores en biología acerca de la ciencia
}

Lisel-Irina Silvestri, Miriam-Liset Flores y Aníbal-Roque Bar

\section{RESUMEN}

En el marco de estudios concernientes a la conformación de creencias sobre la ciencia en ámbitos de formación disciplinar, se indagan las perspectivas de estudiantes de grado y jóvenes investigadores en biología, para examinar cómo y cuánto podrían modificarse las creencias de estos últimos, dado el escaso tiempo transcurrido entre la finalización de la formación de grado y el inicio de trayectorias de investigación en posgrado. Así, se pretende caracterizar y comparar sus perspectivas acerca del conocimiento científico. Se aplicó un cuestionario semiestructurado que incluyó planteamientos precategorizados y una tarea para la construcción de explicaciones en torno de: a) naturaleza de la ciencia, b) operaciones sustanciales del quehacer científico, c) propiedades de las teorías científicas y d) atribuciones sobre la observación. El análisis de los resultados muestra que lo construido en las praxis disciplinares se explicita en una imagen de ciencia donde prima una ontología objetivista y una epistemología ciertamente relativista.

Palabras clave: ciencia, estudiantes, becarios, formación de investigadores, biología, Argentina.

\section{Lisel-Irina Silvestri}

Argentina. Maestra en Psicología Cognitiva y Aprendizaje, FLACSO, Argentina, Profesora y Licenciada en Ciencias de la Educación por la Universidad Nacional del Nordeste (UNNE), Argentina. Jefe de Trabajos Prácticos Regular y Secretaria Técnica del Instituto de Investigaciones en Educación, Facultad de Humanidades, UNNE, Argentina. Temas de investigación: psicología educacional, formación y aprendizaje universitario, estrategias y estilos de aprendizaje, creencias epistemológicas de investigadores en formación.

\section{Miriam-Liset Flores}

miriamliset20@gmail.com

Argentina. Profesora en Ciencias de la Educación, Universidad Nacional del Nordeste (UNNE), Argentina. Auxiliar Docente de primera categoría en la cátedra de Psicología, Becaria Doctoral UNNE-CONICET, Argentina; actualmente, en el marco de la tesis doctoral, se encuentra examinando aspectos referidos a la formación para la investigación disciplinar en las carreras de Biología y Ciencias de la Educación. Temas de investigación: formación disciplinar, formación para la investigación.

\section{Aníbal-Roque Bar}




\title{
A perspectiva de estudantes e jovens pesquisadores em biologia sobre a ciência
}

\section{RESUMO}

No marco de estudos relativos à conformação de crenças sobre a ciência em âmbitos de formação disciplinar, se indagam as perspectivas de estudantes do ensino superior e jovens investigadores em biologia, para examinar como e quanto poderiam modificar-se as crenças destes últimos, dado o escasso tempo transcorrido entre a finalização da formação do curso e o início de trajetórias de pesquisa em pós-graduação. Assim, pretende-se caracterizar e comparar suas perspectivas sobre o conhecimento cientifico. Se aplicou um questionário semiestruturado que incluiu abordagens pré-categorizadas e uma tarefa para a construção de explicações em torno de: a) natureza da ciência, b) operações substanciais do trabalho cientifico, c) propriedades das teorias cientificas e d) atribuições sobre a observação. A análise dos resultados mostra que o construído nas praxis disciplinares se explicita em uma imagem de ciência onde prima uma ontologia objetivista e uma epistemologia certamente relativista.

Palavras chave: ciência, estudantes, bolsistas, formação de pesquisadores, biologia.

\section{The perspective of students and young researchers in biology about science}

\begin{abstract}
In the framework of studies about the formation of beliefs about science in areas of disciplinary training, this study does research into the perspectives of undergraduate students and young researchers in Biology, in order to examine how and how much the beliefs of the latter could be modified, given the short time that has elapsed between the completion of undergraduate training and the beginning of graduate research trajectories. Doing so, this research intends to characterize and compare their perspectives on scientific knowledge. A semi-structured questionnaire was applied that included pre-categorized approaches and a task for the construction of explanations about: a) the nature of science, b) the substantial operations of the scientific task, c) the properties of scientific theories and d) the attributions on observation. The analysis of the results shows that what has been constructed in the disciplinary praxis is made explicit in an image of science in which an objectivist ontology and a certainly relativistic epistemology prevail.
\end{abstract}

Key words: science, students, scholars, researcher training, biology. 


\section{Introducción}

El artículo se enmarca en los estudios concernientes a la conformación de creencias en ámbitos de formación disciplinar, particularmente de estudiantes ${ }^{1}$ y becarios de investigación en biología, acerca de dimensiones que refieren a la ciencia como conocimiento constituido y como proceso de producción de conocimiento. Quienes se forman en sus respectivas disciplinas internalizan y recrean creencias de modo intuitivo a través de su participación en la dinámica de praxis académicas formativas. En este marco interesa recuperar la singularidad del contexto de la biología, ciencia monoparadigma en la que tienen lugar las praxis de los sujetos mencionados. Para Biglan (1973), este tipo de campos disciplinares se estructuran en torno a un mismo cuerpo teórico de conocimiento, a diferencia de aquellos donde se puede abordar un problema común desde diversas perspectivas teóricas, como las ciencias sociales. Al referir a la naturaleza diferenciada de este tipo de disciplinas, Marciales Vivas (2003) señala que las disciplinas monoparadigma son políticamente más conservadoras, se encuentran más comprometidas con la investigación y priorizan el aprendizaje de datos y conceptos. Particularmente, en la biología se destaca la presencia de cuerpos teóricos acordados y con pretensión de universalidad para dar cuenta de los fenómenos, cuya capacidad explicativa y predictiva se apoya en dominios empíricos estrictamente delimitados. Los cánones metodológicos, fuertemente pautados con miras a la justificación del conocimiento, se encuentran en consonancia con el amplio margen de asertividad de los enunciados científicos (Corral y Bar, 2012). Según los autores, si bien los procesos de justificación poseen un doble anclaje igualmente relevante, lógico y empírico, éste último tiene un valor agregado en razón de que concede la evidencia observacional que contribuye a la objetividad.
La construcción de conocimiento biológico y su justificación no se inicia en el laboratorio o en el campo, sino en instancias formativas dadas en el entorno académico. $\mathrm{Al}$ respecto, resulta pertinente la propuesta de Echeverría (1998), quien redefine la clásica distinción sobre los contextos de descubrimiento y justificación de Hans Reichenbach. Describe cuatro contextos para explicar la dinámica tecnocientífica: educación, innovación, evaluación y aplicación; entre los cuales resultan de interés para nuestra propuesta los tres primeros.

Los estudiantes se hallan inmersos en el contexto de educación, vinculados plenamente con los ámbitos de enseñanza y aprendizaje de la ciencia. En el trayecto de la formación inicial, conforman un modelo de ciencia que deviene de lo que Kuhn (1971) denominó ejemplares compartidos, es decir, aquellas soluciones exitosas que operan como guías prácticas para la resolución de problemas concretos aceptados por la comunidad científica como paradigmáticos. El uso y desarrollo de estos ejemplares no sólo conlleva una forma de entender la naturaleza de la disciplina, sino también ciertos modos de interrogar la realidad y de abordar su estudio.

Los becarios de investigación, aunque jóvenes recientemente egresados, ya han abandonado el contexto educativo para introducirse en los de innovación y evaluación, si bien podría preverse que es este último el que tendrá primacía, dada su escasa experiencia en el campo de la investigación. En lo que refiere a la figura del becario de investigación, Aguirre (2013) aporta una caracterización general en un trabajo reciente acerca de las trayectorias de formación en investigación en el campo de las ciencias naturales. En dicho estudio, lo describe como un egresado de carreras de grado, de una duración mínima de cuatro años que, a través de convocatorias de periodicidad anual, accede a trayectos de beca

${ }^{1}$ Saberes y prácticas cognitivas en el contexto de formación disciplinar en biología. Su contribución al logro de ciudadanía. PI: H006/11, acreditado por la Secretaría General de Ciencia y Técnica, UNNE. Proyecto concluido. 
consistentes en la realización de trabajos de investigación y/o desarrollo orientados a la generación y transferencia de conocimientos científicos, tecnológicos y humanísticos. Estos trabajos pueden ser utilizados para la elaboración de una tesis doctoral, condición básica para quienes pretendan ingresar a la carrera de investigador científico del Consejo Nacional de Investigaciones Científicas y Técnicas (CONICET, Argentina). Según la autora, los trayectos de este tipo de beca involucran un complejo desafío, a saber: llevar adelante un proyecto de investigación sobre un tema generalmente muy específico que implica la adquisición de nuevos conocimientos teóricos y el aprendizaje de diversas técnicas; incorporarse progresiva y activamente tanto a un equipo de investigación particular como a una comunidad disciplinar más amplia, y por último, conquistar y sostener el control frente a las vicisitudes de las nuevas situaciones académicas, laborales e interpersonales que surgen en el seno de los equipos de investigación.

Teniendo en cuenta la especificidad del hacer y de los trayectos formativos realizados por ambos sujetos, vale interrogarse sobre cuáles son los rasgos que configuran el contenido de las creencias sobre la ciencia que han conformado los estudiantes durante la formación inicial; del mismo modo, y atendiendo a las nuevas trayectorias de los becarios, si es posible reconocer matices en el contenido de sus creencias respecto de las sustentadas por los alumnos.

Conforme lo expuesto, un primer objetivo es caracterizar las creencias sobre la ciencia que sustentan estudiantes y becarios, centrando el análisis en cuatro dimensiones solidarias entre sí: a) la naturaleza de la ciencia, b) las operaciones sustanciales sobre las cuales se construye y desarrolla el quehacer científico, c) las propiedades de las teorías y d) el papel de la observación en la producción del conocimiento. Como fue mencionado, unos y otros comparten una formación disciplinar común en lo que hace a la carrera de grado, pero los jóvenes investigadores han recorrido además diversos trayectos formativos para la investigación en biología, lo cual introduce aprendizajes y experiencias singulares a partir de las cuales pudieron haber recreado las creencias constituidas en el curso de la formación inicial. En consecuencia, un segundo objetivo es comparar sus creencias respecto de aquellas que revelan los estudiantes y examinar posibles matices en sus contenidos

\section{Antecedentes}

En el ámbito de la psicología, el concepto creencias coexiste con nociones afines tales como concepciones o teorías implícitas, e incluso en algunos trabajos estos términos se utilizan indistintamente, sin especificaciones sobre sus alcances y rasgos definitorios. A pesar de la falta de acuerdo, gran parte de las conceptualizaciones refieren a un tipo de saber cuya validez no se discute, lo cual supone que, al menos en principio, no es pasible de modificación inmediata, ni está abierto a la redefinición de sus connotaciones. Así, Lipman (1997) define a las creencias como pensamientos sobre cuya validez se está convencido, no obstante la ausencia de reflexión en cuanto a su valor de verdad.

Más específicamente, las creencias epistemológicas conciernen a las formas en que se genera, valida y evoluciona el conocimiento científico. De acuerdo con Pérez Echeverría et al. (2006), las personas desarrollan y sustentan una epistemología personal acerca de la naturaleza del conocimiento que está influida, y a la vez influye, en el modo en que nos aproximamos y reflexionamos sobre el mismo.

Ciertas investigaciones las diferencian en tres niveles: generales, académicas y disciplinares (Buehl y Alexander, 2001, 2006; Hofer, 2006). Se reconoce en estos hallazgos un carácter progresivo de lo general a lo particular, donde las creencias a nivel disciplinar se derivarían progresivamente desde las generales, conforme avanza la escolarización (Leal Soto, 2010). En estudiantes de carreras universitarias cabría esperar un predominio de creencias académicas y disciplinares por sobre las generales. El autor afirma 
que la generalidad o especificidad de las creencias a través de las disciplinas o dominios de conocimiento es precisamente uno de los tópicos que aún permanece confuso. La discusión se organiza en torno de si hay o no una estructura generalizada a través de los dominios. Frente al carácter no concluyente de las evidencias disponibles, se ha argumentado que probablemente estas creencias sean en algún grado específicas y en algún grado generales. La orientación de los instrumentos utilizados en los estudios podría explicar las diferencias en los resultados reportados.

Si bien no puede afirmarse que la totalidad de las creencias examinadas en este trabajo hayan sido forjadas durante la formación, sí puede alegarse que en su mayor parte poseen el rango de académicas y/o disciplinares y, en este sentido, deberían guardar una relación más o menos estrecha con la concepción de biología operante en la formación.

Como construcciones sociales, las creencias epistemológicas se conformarían en la interacción con el contexto educativo (Hofer y Pintrich, 1997; Vázquez Alonso et al., 2010). Los contenidos dados en la educación se relacionan en mayor o menor medida con conceptos de raigambre disciplinar, independientemente de su diversidad. Los contextos educativos, ligados con el grado de escolarización, operan con distintos niveles de nociones y teorías, algunos como meros dominios de conocimiento, otros como verdaderos conocimientos disciplinares. Ambos refieren al conocimiento declarativo, procedimental y condicional sobre un campo particular, pero se diferencian en su extensión y organización, necesariamente más complejos en el conocimiento disciplinar (Alexander, 1992). Según Hofer et al. (1997), el uso indistinto de los términos dominio de conocimiento y conocimiento disciplinar plantea dificultades al momento de precisar cómo inciden en la estructuración de estas creencias.

Diversos autores sostienen que las creencias de los estudiantes se conforman de distintas perspectivas epistemológicas, muchas de ellas contradictorias entre sí, cuestión que no es advertida como tal ni produce conflicto alguno (Petrucci y Dibar Ure, 2001; Vázquez Alonso et al., 2010).

Los hallazgos de Thomaz et al. (1996) revelaron la primacía de una visión dinámica del conocimiento científico en futuros profesores de enseñanza primaria, quienes en su mayoría destacaron el carácter temporal de las teorías. Señalaron también la marcada presencia de una perspectiva empirista sobre la construcción del conocimiento y de ideas inductivistas acerca del método científico, las cuales enfatizan en la observación como punto de partida para el desarrollo del conocimiento. Para Petrucci et al. (2001), los alumnos universitarios de biología y geología, aunque en una versión ingenua, acuerdan en que las teorías se reemplazan o corrigen como mera sustitución.

Briceño Martínez y Benarroch (2013) realizaron tests y entrevistas semiestructuradas a profesores universitarios de ciencias para examinar sus creencias respecto de la naturaleza de la ciencia, el aprendizaje científico y su enseñanza. Concluyeron acerca del predominio de concepciones empiristas frente a las constructivistas, concepciones reduccionistas sobre la enseñanza y un relativo desconocimiento del proceso de aprendizaje.

Se ha afirmado que docentes universitarios de biología adhieren al relativismo en la convicción de que no existen verdades universalmente válidas, $\mathrm{y}$ conciben la esencia del conocimiento desde una postura realista crítica (García et al., 2011). En esta línea, Acevedo Díaz (2000) sostiene que los profesores de biología conciben una ciencia cambiante en virtud de cierto contexto histórico, lo cual no impide que la realidad pueda abordarse con alguna exactitud en pos de la objetividad.

Por el contrario, Rabanal Moreno y Quintanilla Gatica (2010) hallaron que las concepciones epistemológicas de estos profesores son de carácter absolutista, a la vez que centradas principalmente en la justificación del conocimiento, más que en su producción y valoración. Consideran que los docentes 
de esta disciplina no han logrado desvincularse de una imagen de ciencia con vertientes de índole empirista o racionalista.

Alvarado Rodríguez y Flores-Camacho (2001) exploraron las concepciones de ciencia y de su enseñanza en docentes investigadores de una universidad mexicana, entre cuyas tareas prioritarias destaca la actividad científica en centros especializados de física, química, matemática y biología. Sus principales hallazgos ponen de relieve el notorio desconocimiento sobre cuestiones históricas, contextuales, epistemológicas y educativas por parte de estos académicos, lo que explica la escasa atención concedida a la investigación en el campo de la enseñanza de la ciencia y las características poco alentadoras que definen el modelo de enseñanza en dicho contexto. De la Lama García et al. (2013) han explorado la cultura científica mediante un sondeo de opinión concluyendo que, independientemente de las disciplinas, los investigadores reconocen explícitamente cuatros reglas de juego: la captación del mundo mediante la observación, la garantía del método para poner en consideración los puntos de partida, la actitud crítica durante todo el proceso de indagación y la comunicación de resultados sustentada en metodologías verificadoras.

Estudios como los hasta aquí reseñados sugieren un amplio desarrollo en la indagación de las creencias epistemológicas de estudiantes y profesores; sin embargo, aún son escasos los antecedentes que sitúan el análisis en la perspectiva de investigadores de distintas áreas disciplinares.

\section{Decisiones metodológicas}

El trabajo tiene finalidad heurística y se orienta en la búsqueda de las creencias sobre la ciencia detentadas por estudiantes y becarios de biología. Se privilegió un tratamiento de naturaleza cualitativa, asumiendo que las cantidades y frecuencias relevadas sólo mostrarían tendencias, sentidos mayoritarios y concepciones compartidas. El diseño es transversal, en tanto la información fue recabada en un tiempo único.

\section{Participantes}

En el ámbito de la formación inicial y de posgrado en biología se definieron dos muestras intencionales. Una de ellas conformada por 18 estudiantes $(\mathrm{n}=18)$ cursantes de los últimos niveles de la carrera, y la otra, por 25 egresados $(n=25)$ que desarrollaban sus respectivos proyectos en el marco de becas de investigación.

En el primer caso, se seleccionaron la totalidad de los alumnos que se encontraban cursando una asignatura de cuarto año y tenían el ciclo básico aprobado, criterio de inclusión que se definió sobre el supuesto de que conocían debidamente los contenidos específicos y las operaciones que la disciplina inculcó durante la formación.

En el segundo, se seleccionaron becarios de posgrado que desempeñaban sus actividades en distintos departamentos/cátedras de la universidad y en institutos dedicados a la investigación en ciencias biológicas. Se trata de investigadores en instancia de formación doctoral cuyos trayectos se hallan institucionalizados en el ámbito del sistema de ciencia y técnica regional, lo cual asegura por un lado la accesibilidad, y por otro, su idoneidad como informantes clave respecto del tema. En todos los casos atraviesan un doble proceso de selección para eventualmente incorporarse a grupos de investigación. El primero, de carácter más informal, dado que usualmente los docentes-investigadores los invitan a integrar equipos de cátedra como pasantes o adscritos. El segundo proceso, de índole formal, para acceder al sistema de becas mediante la postulación a convocatorias anuales en las que sus antecedentes son evaluados por comisiones de investigadores consolidados; todo lo cual permite conjeturar que se trata de jóvenes con aptitudes y cualidades para su desarrollo profesional en la actividad tecnocientífica. Los sujetos fueron debidamente informados acerca de los propósitos de la investigación y el tipo de información requerida, y en todos los casos accedieron a participar de forma voluntaria. 


\section{Instrumento}

Se diseñó un cuestionario semiestructurado elaborado ad hoc sobre distintas dimensiones referidas a la ciencia como conocimiento constituido y como proceso de conocer. Las cuestiones seleccionadas para esta publicación, se expresaron en ítems que denotan matices sobre el conocimiento, sin la intención de adscribir a los sujetos a un modelo de ciencia de la tradición epistemológica, sino más bien orientado a denotar cuál concepción de biología es la imperante entre los participantes.

En lo que sigue, los ítems elaborados para cada dimensión se presentan en cuadros con sus respectivas opciones de respuesta, tal como fueron propuestos en el protocolo.

- Naturaleza de la ciencia. Se ofrecieron diferentes concepciones para escoger aquella que mejor reflejara la peculiaridad de la ciencia. En dos de ellas la prioridad corresponde a la observación, aunque con matices diferenciados; en otra se enfatiza el carácter convencional de las reglas generadas en las comunidades científicas; en las otras dos la preeminencia recae en la teorización, aunque con variaciones particularizadas. Complementariamente, se incluyó una instancia de elaboración libre para justificar la selección.

Te presentamos cinco concepciones que ilustran el carácter de la ciencia haciendo énfasis en distintas propiedades. Selecciona sólo una, la que mejor refleje la imagen que te haces de ella, marcando la opción con una cruz. La ciencia es ante todo...

a) Registro de los datos que describen los fenómenos y su evolución.

b) Transformación de información para mostrar estados uniformes del mundo y para encontrar regularidad bajo la aparente irregularidad.

c) Un sistema de reglas, construido y acordado convencionalmente por los científicos para elaborar explicaciones útiles.

d) Un sistema teórico para dar respuestas a nuestros cómo y a nuestros por qué sobre el funcionamiento de lo real.

e) Un sistema de conjeturas para resolver interrogantes con los datos de la experiencia. ¿Qué te induce a pensar que la concepción escogida refleja mejor que las otras tu imagen de la ciencia?

- Operaciones sustanciales del quehacer científico. Se plantearon diversas operaciones, con la aclaración de que la actividad científica se apoya al mismo tiempo en todas ellas, pero con predominio de alguna según los momentos históricos y las disciplinas.

Aunque la ciencia marcha, al mismo tiempo, sobre "cuatro pilares", en distintos momentos históricos y en diferentes campos disciplinares, una de ellas predomina. En tu ámbito de formación, ¿cuál de estos pilares crees que es el más importante? Marca tu opción con una cruz.
a) La teorización
b) La observación
c) La imaginación
d) La verificación

- Propiedades de las teorías cientificas. Se propusieron cuatro pares dicotómicos de definiciones para caracterizarlas. Conforme su proximidad o afinidad con la posición personal, se indicó escoger una alternativa por cada par.

Te proponemos parejas de definiciones que aluden a las teorías. Para cada par debes seleccionar una de ellas, marcando con una cruz la opción correspondiente de acuerdo con tu perspectiva. Las teorías científicas son... 
Primer par

a) Conjuntos de afirmaciones derivadas de la observación.

b) Especulaciones para ser confrontadas con la observación.

Segundo par

a) Respuestas definitivas a los interrogantes sobre lo real.

b) Conjeturas aquí y ahora.

Tercer par

a) Aproximaciones a la realidad objetiva.

b) Formas históricas y culturales de concebir el mundo.

Cuarto par

a) El modo más fiable de dar cuenta de los hechos.

b) Una manera de dar cuenta de los hechos, entre otros modos posibles.

- Atribuciones de la observación en los procesos científicos. Se consideraron dos planteamientos precategorizados; uno refiere al lugar asignado a la observación en el vínculo entre sujeto y objeto, y el otro a su posibilidad de aproximarse a lo real o de rescatar las cualidades del objeto. En ambos, se recurrió a metáforas que ilustraban las propiedades de la observación en las dos acepciones invocadas.

Nos ocupamos aquí de la observación en los procesos científicos. Elije, marcando con una cruz, la imagen que mejor exprese tu pensamiento sobre lo más decisivo en la índole de la observación científica. La observación es...

a) La roca firme sobre la que se construye el conocimiento.

b) El tribunal último que decide el valor de las teorías.

c) El intérprete entre los datos sensoriales y los conceptos.

d) El resultado de las prácticas arraigadas en las comunidades científicas.
Por último, marca con una cruz la metáfora que te parece representa mejor el procedimiento de la observación. La observación científica es como...

a) Una fotografía que retrata fielmente el objeto.

b) Una pintura 'clásica' que representa lo observado.

c) Una pintura 'impresionista' que representa el momento observado, aunque los trazos sean difusos.

d) Una pintura 'abstracta' que representa una idea sin referencia figurativa con lo observado.

Los tres primeros ítems implicaron la selección de alternativas que señalaban caracterizaciones generales o aspectos particulares de la ciencia. La elección de cada tópico requería acudir a la literalidad del enunciado, pues no implicaba interpretaciones complejas ni procesos mediacionales.

Para el cuarto ítem se seleccionaron metáforas que evocaban algún elemento de la ciencia. El uso de este recurso se funda en que ésta asume un rol cognitivo lingüístico en la comprensión de la realidad al ofrecer correspondencias entre un dominio fuente y un dominio meta, donde los elementos conceptuales de uno se relacionan con los elementos conceptuales del otro (Santibañez, 2009). Lakoff y Johnson (1980) denominan mapeo a este proceso, esto es un concepto puesto en la cotidianidad del lenguaje. Para estos autores, lo experiencial juega un rol esencial, toda vez que los dominios fuente se enraízan en lo cotidiano y en aquello más próximo a la experiencia corpórea, en tanto que los dominios meta son de naturaleza más abstracta y llevan consigo los sentidos transferidos desde éstos. Así, las metáforas conceptuales muestran mapeos entre dominios, pero no de cualquier modo, pues son selectivas, o sea, resaltan u ocultan los diversos aspectos de la experiencia (Kövecses, 2002). 


\section{Procedimientos de análisis}

La caracterización de las creencias se desarrolló a partir del análisis de los datos obtenidos en las elecciones y respuestas libres de los sujetos, buscando identificar propiedades generales e indicativas de identidad disciplinar. Para su tratamiento se determinó la frecuencia de las respuestas precodificadas, intentando vincular los señalamientos con las perspectivas del marco referencial y el conocimiento del contexto disciplinar. En la instancia complementaria del ítem sobre las concepciones de ciencia, se procedió a interpretar los sentidos y énfasis emergentes en las justificaciones de las elecciones, para reconocer posicionamientos diferenciados y examinar su consistencia con respecto a las opciones elegidas.

En lo atinente a las metáforas se acudió a Fabbri (1999) para su interpretación, pues la sitúa en el mismo nivel que la inferencia, al asimilar ambas al incremento o extensión del conocimiento, postura que se opone a la antigua tradición que discrimina taxativamente las figuras retóricas u ornamentos discursivos de los rigurosos silogismos y sus demostraciones deductivas. Para la construcción de inferencias desde las metáforas, se utilizó la propuesta de Giacaglia (2007), para quien los aportes de Fabbri y de Samaja (1993) contribuyen a entender la metáfora como tomando cuerpo en el proceso de investigación en una secuencia de tres fases, a las que acudimos para el análisis: 1) constitución de vínculos de correspondencia analógica entre el fenómeno - la observación - y un determinado esquema de narración; 2) integración de las analogías en un sistema de mayor nivel vía abducción desde los indicios; y 3) elaboración de un esquema inferencial complejo construido desde un lenguaje figurado.

En la secuencia anterior, la abducción se expresó como la herramienta más importante para "hacer hablar" a la metáfora, y en este contexto, constituyó un invalorable aporte metodológico a la reconstrucción de esquemas o conceptos. Como último procedimiento de análisis, se compararon e integraron los datos relativos a las muestras.

\section{Discusión de resultados}

El sistema de creencias construido por los estudiantes y los becarios en el curso de sus praxis formativas, muestra rasgos solidarios en los sentidos que connotan las dimensiones indagadas.

El análisis de los resultados se organiza en función de las selecciones realizadas por cada muestra de sujetos para las diversas tareas, las cuales se sintetizan en tablas de distribución de frecuencia que expresan los valores absoluto y relativo. Para la primera tarea, se incluyen ejemplificaciones de las justificaciones ofrecidas para dar cuenta de la concepción de ciencia privilegiada. Se utilizan los códigos "E" y "B" para referenciar las respuestas de estudiantes y becarios, y en cada caso el código va acompañado de un número que individualiza a los sujetos.

\section{Naturaleza de la ciencia}

En la mayoría de los estudiantes y los becarios prevalece la idea de la ciencia como un sistema de reglas. En lo relativo a las otras opciones, los estudiantes privilegian las concepciones cuyos énfasis recaen en la teorización y en menor proporción, aquellas que priorizan la observación. Entre los becarios, tuvieron mayor aceptación las alternativas que destacaban propiedades vinculadas con la empiria, siendo minoritarias las elecciones que acentuaban el papel de la teoría. En la situación de estos últimos se observa una mayor variabilidad en los señalamientos, lo que podría explicarse en razón de su inserción en praxis de investigación diferenciadas por la especificidad de las subdisciplinas, lo que habilitaría un reconocimiento de distintas interpretaciones o "versiones" sobre la naturaleza de la ciencia. Las elecciones en uno y en otro caso se presentan en la tabla 1.

La ciencia como sistema de reglas pretendió expresar una visión convencionalista de la misma. Esta forma de entender la cuestión pone en valor tres componentes del quehacer científico: la construcción, el acuerdo y la utilidad. El primero sugiere que el conocimiento científico, independientemente de su 
objetividad, es un producto social y en este sentido, deviene de actos que, aunque conscientes, se sustentan en creencias o saberes atravesados por experiencias, tanto personales como colectivas. De igual modo, el acuerdo supone intersubjetividad social y, por lo tanto, conflictos, negociaciones e intereses.
Respecto a la utilidad, ésta no involucra exclusivamente aspectos prácticos, ya que la ciencia por sí puede resolver también problemas de conocimiento sin implicaciones directas en el mundo social, pero sí en el sistema científico.

Tabla 1. Elecciones de estudiantes y becarios para cada concepción de ciencia

\begin{tabular}{|c|c|c|c|c|}
\hline \multirow{2}{*}{$\begin{array}{l}\text { Opciones } \\
\text { Registro de los datos que describen los fenómenos y su evolución. }\end{array}$} & \multicolumn{2}{|c|}{$\begin{array}{l}\text { Estudiantes } \\
\qquad(n=18)\end{array}$} & \multicolumn{2}{|c|}{$\begin{array}{l}\text { Becarios } \\
(n=25)\end{array}$} \\
\hline & 4 & $22 \%$ & 4 & $16 \%$ \\
\hline $\begin{array}{l}\text { Transformación de información para mostrar estados uniformes del mundo y para encontrar } \\
\text { regularidad bajo la aparente irregularidad. }\end{array}$ & 0 & $0 \%$ & 4 & $16 \%$ \\
\hline $\begin{array}{l}\text { Un sistema de reglas, construido y acordado convencionalmente por los científicos para elaborar } \\
\text { explicaciones útiles. }\end{array}$ & 8 & $45 \%$ & 11 & $44 \%$ \\
\hline $\begin{array}{l}\text { Un sistema teórico para dar respuestas a nuestros cómo y a nuestros por qué sobre el funcionamiento } \\
\text { de lo real. }\end{array}$ & 4 & $22 \%$ & 2 & $8 \%$ \\
\hline Un sistema de conjeturas para resolver interrogantes con los datos de la experiencia. & 2 & $11 \%$ & 4 & $16 \%$ \\
\hline TOTALES & 18 & $100 \%$ & 25 & $100 \%$ \\
\hline
\end{tabular}

Fuente: elaboración propia.

Cuando los estudiantes dan cuenta de esta opción, lo hacen sobre todo relacionándola con la normatividad que el sistema de reglas impone, más que con los aspectos convencionales o prácticos. Lo que se rescata de la definición es que a pesar de la subjetividad que suponen los acuerdos, prima lo que emerge de ellos, principios normativos que ya no admiten discusiones posteriores, al menos no en el corto plazo. Los siguientes ejemplos ilustran dicha perspectiva:

La comunidad científica hace ciencia bajo un sistema de estudios, análisis y resultados que todos deben seguir, cumpliendo las reglas (E3).

La ciencia tiene reglas preestablecidas y todos aquellos que hacen ciencia deben acatarlas para que sus conocimientos sean 'aprobados' por los demás científicos (E17).
Lo pragmático no reaparece en las respuestas, con excepción del caso de un alumno que lo refiere en términos de utilidad de las explicaciones que posibilita la actividad científica.

Si bien la idea de la ciencia como transformación de información involucra también aspectos como la regularidad y la legalidad, su no elección por parte de los estudiantes sugiere que estas particularidades no son suficientes per se para caracterizar a la ciencia, pues además se requiere de una comunidad científica que las discuta y ponga en vigencia a posteriori de los debates.

Las concepciones de ciencia como mero registro, o como la teorización que se establece a partir de nuestras inquietudes, o como conjeturas sobre la realidad han tenido menor aceptación. Una explicación posible quizás reside en que la primera constituye una versión acotada y mezquina, la segunda se queda en la mera 
especulación, y la tercera se orienta exclusivamente a la resolución de interrogantes.

Entre quienes optaron por la ciencia como registro de datos sobre los fenómenos y su evolución, sólo dos respondieron consistentemente vinculando su elección con la justificación: "Todos los trabajos científicos se basan en el registro en tablas, gráficos de datos que dan cuenta de sucesos..." (E14).

Una de las respuestas no evidencia relación con el sentido de la alternativa elegida, y la otra, justifica la elección por la ausencia de conformidad respecto de las demás opciones.

Entre los que seleccionaron la concepción de la ciencia como sistema teórico, tres de ellos enfatizan en el papel de las inquietudes que movilizan la actividad científica y las respuestas que puede proveer la ciencia, y sólo uno en el hecho de que la producción de teoría se asimila a la ciencia, no a otra cosa.

Porque creo es lo que hace la ciencia, se basa en encontrar respuestas y brindarlas a la sociedad de manera que todas las inquietudes sean, en la posibilidad, resueltas (E12).

Las justificaciones para la proposición que define la ciencia en términos de un sistema de conjeturas, denotan la idea de adentrarse en la acción investigativa y de las experiencias vividas en ella.

En lo que respecta a los becarios, cuando justifican la elección del enunciado que plantea la ciencia como un sistema de reglas, la mitad focaliza en el componente metodológico para la producción y la valoración de los conocimientos. Lo central en sus apreciaciones son las referencias explícitas o implícitas a la autoridad normativa de los procedimientos metodológicos y a las recomendaciones instrumentales.

$\mathrm{Al}$ pensar la ciencia, imagino una serie de pasos que el investigador debe seguir (pasos del método científico) que los hace generalmente de forma convencional (B6).
La ciencia sólo puede ser definida bajo el marco del método científico, el cual es un sistema, si bien no de reglas, sí de 'pasos' a seguir, y el cual fue convencionalmente acordado por los científicos (B7).

Otro conjunto de respuestas pone de relieve el potencial explicativo del sistema de reglas que conforma la ciencia.

Porque la ciencia basándose en un sistema de reglas, trata de percibir y explicar desde lo esencial hasta lo más complejo, el porqué de las cosas y su devenir (B21).

Dentro de cada campo científico hay reglas acordadas por los científicos y el fin de la ciencia es encontrar posibles explicaciones útiles a ciertas preguntas o interrogantes planteados por la comunidad y la sociedad actual (B23).

Las dos concepciones que priorizan la observación tuvieron una aceptación menor y equivalente. Una de ellas supone la idea de una ciencia que reposa sobre la objetividad del dato con fines descriptivos, en tanto que la otra agrega mención explícita a operaciones de elaboración o manipulación de la información. Las explicaciones de dichas selecciones, ubicaron la observación en el inicio de los procesos de indagación: el registro de datos, ya sea en el laboratorio o en situación natural, lo que permite seguir y comprender la evolución de los fenómenos, como de igual modo posibilita descripciones indispensables como paso previo para la explicación. En este grupo, todas las respuestas especifican operaciones de análisis o de interpretación, como lo evidencian estos ejemplos:

El hombre hace ciencia cuando intenta explicar los fenómenos que ocurren constantemente. Pero primero debe haber una descripción de dicho fenómeno (esto incluye la toma de datos sobre su evolución), para luego ser comprendido como tal (B12). 
Se trabaja en principios con datos para la descripción de ciertos fenómenos y a partir de ahí poder interpretar su evolución (B11).

La caracterización de la ciencia como un sistema de conjeturas para resolver interrogantes con los datos de la experiencia, acentúa en el interjuego entre hipótesis y experiencia. Sin embargo, dos de los cuatro becarios que la eligieron la han connotado desde una versión más empirista, reforzando el papel de la experiencia y de los datos que ella provee como base para la formulación de conjeturas y la producción de teoría. Otro situó su justificación en el camino inverso, atribuyendo a la experiencia la función de poner a prueba la teoría. Considerando los argumentos dados por este grupo respecto de su elección, puede decirse que al connotar el carácter de la ciencia privilegian un sentido u otro, antes que la recursividad entre hipótesis y experiencia.

Finalmente, la afirmación que entiende la ciencia como sistema teórico fue escasamente seleccionada. Uno de los dos becarios que la eligió ubica los interrogantes como punto de partida para la formulación y contrastación de hipótesis y para la elaboración de teorías, el otro alude en líneas generales al objeto que ocupa a la ciencia sin recuperar el sentido implicado en la definición escogida.

\section{Operaciones sustanciales del quehacer científico}

En proporciones similares, ambos grupos destacan la preeminencia de la observación y la verificación, por sobre la teorización y la imaginación, lo que parece solidario con una versión de ciencia de raigambre empirista como es la biología.

Tabla 2. Elecciones de estudiantes y becarios para las operaciones sustanciales del quehacer científico

\begin{tabular}{|l|c|c|c|c|}
\hline Opciones & \multicolumn{2}{|c|}{$\begin{array}{c}\text { Estudiantes } \\
(\mathbf{n = 1 8})\end{array}$} & \multicolumn{2}{c|}{$\begin{array}{c}\text { Becarios } \\
(\mathbf{n}=\mathbf{2 5})\end{array}$} \\
\hline La teorización & 4 & $22 \%$ & 2 & $8 \%$ \\
\hline La observación & 6 & $34 \%$ & 12 & $48 \%$ \\
\hline La imaginación & 1 & $5 \%$ & 1 & $4 \%$ \\
\hline La verificación & 7 & $39 \%$ & 10 & $40 \%$ \\
\hline \multicolumn{1}{|c|}{ TOTALES } & $\mathbf{1 8}$ & $\mathbf{1 0 0 \%}$ & $\mathbf{2 5}$ & $\mathbf{1 0 0 \%}$ \\
\hline
\end{tabular}

Fuente: elaboración propia.

En sus elecciones, la teorización se ubica en un segundo plano y la imaginación casi no es señalada, lo cual podría dar cuenta de una formación que favorece poco la especulación y las elucubraciones carentes de fundamento empírico.

Coincidentemente, los becarios privilegiaron la observación y la verificación como bases de la actividad científica en su disciplina. En la medida en que observar permite también problematizar, plantear hipótesis, describir el objeto, descubrir regularidades, etcétera; puede decirse que mientras unos entienden la observación de modo más amplio y a la verificación como uno de sus potenciales, otros la piensan en términos de la contrastación o 
corroboración de hipótesis. Prácticamente no fueron reconocidas ni la teorización ni la imaginación.

\section{Propiedades de las teorías científicas}

Entre los estudiantes y los becarios la concepción recurrente es que las teorías constituyen conjeturas en un momento dado y que no obstante su naturaleza provisoria, asumen como referencia concreta a la realidad objetiva.

Cuando lo que se pone en juego es el papel de la observación en relación con la teoría, emergen posiciones diferenciadas. Algunos la entienden desde una perspectiva inductivista como el punto de partida para teorizar, otros como la instancia que confronta el plano teórico con lo real, quizás porque el estudio de los fenómenos biológicos implica diversos estados del arte y distintos niveles de empiria, lo que conlleva a que la comprensión de sus objetos esté más próxima a la modelización teórica, o bien al conocimiento emergente de observaciones más o menos directas.

En cuanto a la confiabilidad de las teorías, las preferencias de los estudiantes se volcaron en igual proporción a pensar la ciencia con un status de saber excluyente en su posibilidad de dar cuenta de los hechos, o como un modo entre otros igualmente legítimos. Algo similar ocurrió con los becarios, hay quienes la ven como la forma más fiable de dar cuenta de la realidad, en tanto otros la relativizan, asimilándola a la idea de alternativa posible pero no única para decir algo acerca del mundo objetivo.

Puede afirmarse que en ambos grupos prima una ontología objetivista, si bien lo epistemológico transita por un camino de cierto relativismo. Que la teoría pueda concebirse tanto como el modo más fiable de dar cuenta de los hechos o como una manera entre otras posibles, puede comprenderse a partir de que algunos se posicionaron en la idea de que la teoría es el tipo de enunciado de mayor complejidad y abstracción, y en este sentido, es más fiable que cualquier otro tipo de discurso. Otros, en cambio, pudieron haber entendido que existen otras formas de dar cuenta de los hechos, como las generalizaciones empíricas o aún los enunciados singulares, todos ellos en vínculo con instancias de producción o justificación del conocimiento biológico.

Tabla 3. Elecciones de estudiantes y becarios para cada propiedad de las teorías

\begin{tabular}{|c|c|c|c|c|c|}
\hline \multicolumn{2}{|l|}{ Opciones } & \multicolumn{2}{|c|}{$\begin{array}{l}\text { Estudiantes } \\
\qquad(n=17)^{2}\end{array}$} & \multicolumn{2}{|c|}{$\begin{array}{c}\text { Becarios } \\
(n=25)\end{array}$} \\
\hline \multirow{2}{*}{ Primer par } & Conjuntos de afirmaciones derivadas de la observación & 9 & $53 \%$ & 15 & $60 \%$ \\
\hline & Especulaciones para ser confrontadas con la observación & 8 & $47 \%$ & 10 & $40 \%$ \\
\hline \multicolumn{2}{|r|}{ TOTALES } & 17 & $100 \%$ & 25 & $100 \%$ \\
\hline \multirow{2}{*}{ Segundo par } & Respuestas definitivas a los interrogantes sobre lo real & 4 & $24 \%$ & 6 & $24 \%$ \\
\hline & Conjeturas aquí y ahora & 13 & $76 \%$ & 19 & $76 \%$ \\
\hline \multicolumn{2}{|r|}{ TOTALES } & 17 & $100 \%$ & 25 & $100 \%$ \\
\hline \multirow{2}{*}{ Tercer par } & Aproximaciones a la realidad objetiva & 13 & $72 \%$ & 21 & $84 \%$ \\
\hline & Formas históricas y culturales de concebir el mundo & 5 & $28 \%$ & 4 & $16 \%$ \\
\hline \multicolumn{2}{|r|}{ TOTALES } & 18 & $100 \%$ & 25 & $100 \%$ \\
\hline \multirow{2}{*}{ Cuarto par } & El modo más fiable de dar cuenta de los hechos & 9 & $53 \%$ & 10 & $40 \%$ \\
\hline & Una manera de dar cuenta de los hechos, entre otros modos posibles & 8 & $47 \%$ & 15 & $60 \%$ \\
\hline \multicolumn{2}{|r|}{ TOTALES } & 17 & $100 \%$ & 25 & $100 \%$ \\
\hline
\end{tabular}

Fuente: elaboración propia.

${ }^{2}$ En este ítem la muestra fue de diecisiete (17) estudiantes, ya que uno de ellos respondió sólo en uno de los pares propuestos (véase tercer par). 
Atendiendo a un segundo nivel de análisis, se observan las combinaciones de señalamientos producidas por los participantes. En este sentido, a partir de combinar los términos de las cuatro parejas de afirmaciones, se definen a priori dos perspectivas diferenciadas:

- La primera se aproxima a una versión más clásica de la ciencia: "Las teorías científicas son conjuntos de afirmaciones derivadas de la observación, constituyen respuestas definitivas a los interrogantes sobre lo real. Se trata de aproximaciones a la realidad objetiva, en consecuencia, son el modo más fiable de dar cuenta de los hechos".

- La segunda se vincula con una orientación constructivista con ciertas evocaciones relativistas: "Las teorías científicas son especulaciones para ser confrontadas con la observación, son conjeturas aquí y ahora, por consiguiente, son formas históricas y culturales de concebir el mundo, y como tales constituyen una manera de dar cuenta de los hechos, entre otros modos posibles".

Entre los estudiantes, dos fueron totalmente consistentes con el posicionamiento de ciencia clásica, y otros dos con la perspectiva constructivista/relativista. Entre los becarios, tres adhirieron la primera y sólo uno a la segunda. Los restantes seleccionaron caracteres mixtos entre ambos modelos; en un caso seleccionando tres de las cuatro dimensiones relativas a uno de los modelos definidos (6 estudiantes y 14 becarios), y en el otro, privilegiando dos de las dimensiones próximas a cada uno de ellos (7 estudiantes y 7 becarios).

Dichas mixturas no implican necesariamente inconsistencia, sino más bien interpretaciones que pueden mostrar compatibilidad con otras formas de comprensión del fenómeno biológico. Por ejemplo, en el grupo de los becarios cuyas elecciones se aproximan a una concepción constructivista-relativista, muchos combinan los rasgos de este modelo con la afirmación de que las teorías son aproximaciones a la realidad objetiva, lo cual parece expresar la fuerte intuición de una realidad independiente del sujeto que la piensa. Aun teniendo carácter conjetural, el conocimiento científico aspira a la aprehensión de lo real.

De igual manera, en el caso de los sujetos cuyas elecciones privilegian una concepción más clásica de la ciencia, la mayoría toma distancia al reconocer la naturaleza provisoria de las teorías en tanto posibles respuestas situadas en tiempo y espacio.

\section{La observación en los procesos científicos Qué es la observación}

Los señalamientos de los participantes se distribuyen básicamente entre dos de las alternativas ofrecidas, como lo muestra la tabla 4.

\section{Tabla 4. Elecciones de estudiantes y becarios sobre la naturaleza de la observación}

\begin{tabular}{|c|c|c|c|c|}
\hline \multirow{2}{*}{$\begin{array}{ll}\text { Opciones } & \text { Sujetos } \\
\text { La roca firme sobre la que se construye el conocimiento } & \end{array}$} & \multicolumn{2}{|c|}{$\begin{array}{l}\text { Estudiantes } \\
\qquad(n=18)\end{array}$} & \multicolumn{2}{|c|}{$\begin{array}{c}\text { Becarios } \\
(n=25)\end{array}$} \\
\hline & 6 & $33 \%$ & 10 & $40 \%$ \\
\hline El tribunal último que decide el valor de las teorías & 1 & $6 \%$ & 0 & $0 \%$ \\
\hline El intérprete entre los datos sensoriales y los conceptos & 9 & $50 \%$ & 14 & $56 \%$ \\
\hline El resultado de las prácticas arraigadas en las comunidades científicas & 2 & $11 \%$ & 1 & 4 \\
\hline TOTALES & 18 & $100 \%$ & 25 & $100 \%$ \\
\hline
\end{tabular}

Fuente: elaboración propia. 
Resulta mayoritariamente privilegiada la opción concerniente a la observación como mediadora entre datos y conceptos, escogida por la mitad de los estudiantes y por poco más de la mitad de los becarios. En ambas muestras, un grupo menor la identifica como la roca firme sobre la que se construye el conocimiento, lo que constituye una versión más próxima a los enfoques clásicos. La escasa elección de las otras imágenes sugiere, por un lado, el carácter limitado de la observación en su posibilidad de dirimir concluyentemente el valor de la teoría, y por otro, que ésta constituye una manera objetiva de vincularse con el conocimiento, y en consecuencia no es sólo el producto de prácticas encarnadas en las instituciones.

\section{Cómo opera la observación en la aprehensión de la realidad}

En ambos grupos las selecciones se concentran en las posiciones intermedias respecto a los sentidos que evocan las metáforas ofrecidas, la pintura clásica o la pintura impresionista. Cualquiera de ellas son sólo representaciones de la realidad, aunque difieren en el nivel de precisión para reflejarla.

Tabla 5. Elecciones de estudiantes y becarios sobre el proceso de la observación

\begin{tabular}{|c|c|c|c|c|}
\hline \multirow{2}{*}{$\begin{array}{ll}\text { Opciones } & \text { Sujetos } \\
\text { Una fotografía que retrata fielmente el objeto } & \end{array}$} & \multicolumn{2}{|c|}{$\begin{array}{l}\text { Estudiantes } \\
\qquad(\boldsymbol{n}=18)\end{array}$} & \multicolumn{2}{|c|}{$\begin{array}{c}\text { Becarios } \\
(n=25)\end{array}$} \\
\hline & 1 & $6 \%$ & 5 & $20 \%$ \\
\hline Una pintura 'clásica' que representa lo observado & 9 & $50 \%$ & 8 & $32 \%$ \\
\hline Una pintura 'impresionista' que representa el momento observado aunque los trazos sean difusos & 8 & $44 \%$ & 12 & $48 \%$ \\
\hline Una pintura 'abstracta' que representa una idea sin referencia figurativa con lo observado & 0 & $0 \%$ & 0 & $0 \%$ \\
\hline TOTALES & 18 & $100 \%$ & 25 & $100 \%$ \\
\hline
\end{tabular}

Fuente: elaboración propia.

Como evidencian los datos, las elecciones de los participantes se distancian tanto del supuesto de que la observación rescata fielmente lo observado (una fotografia), como de aquella concepción que la entiende como un proceso cargado de connotaciones subjetivas (una pintura abstracta). Estas últimas prácticamente no tuvieron aceptación entre los estudiantes, mientras que menos de un tercio de los becarios optó por seleccionar la primera metáfora.

A modo de discusión, pueden referirse algunas similitudes entre el contenido de las creencias examinadas en estos sujetos y los resultados de estudios en otros contextos. En este sentido, el reconocimiento del carácter situado y conjetural de las teorías científicas es acorde con las afirmaciones de Acevedo Díaz
(2000), quien expresa que los profesores de biología conciben una ciencia cambiante en virtud de cierto contexto histórico, lo cual no impide que pueda abordarse la realidad con alguna exactitud en pos de la objetividad. Asimismo, los hallazgos de Thomaz et al. (1996) en estudiantes de profesorado de enseñanza primaria, reportan que normalmente se admite el cambio de teorías y conceptos, lo que implica dotar de un status temporal al conocimiento.

Lo afirmado también es coincidente con lo planteado por García et al. (2011) cuando sostienen que los profesores universitarios de biología adhieren al relativismo en la convicción de que no existen verdades universalmente válidas, y que conciben la esencia del conocimiento desde una postura realista 
crítica, que entiende la realidad como una construcción consensuada por una comunidad en busca de cierta objetividad.

Por otra parte, la observación es entendida como generadora de conocimiento y como mediadora entre la teoría y los hechos, lo cual guarda semejanza general con lo encontrado por estudios que afirman que tanto estudiantes como docentes suponen un doble origen del conocimiento, racional por un lado, empírico por el otro, aunque valorando especialmente este último (Manassero y Vázquez, 2000; García et al., 2011).

\section{Conclusiones}

En sentido amplio, los resultados sugieren que lo construido por los estudiantes y los becarios en el marco de sus praxis formativas se formula explícitamente en términos de una imagen de ciencia de tinte racional-empirista, si bien tangencialmente se filtran elementos que la vinculan con situaciones históricas y contextuales (Bar, 2013). En la perspectiva de este autor, pareciera que la experiencia empírica en primera instancia, y la racional, de modo secundario, constituyen lo que la mayoría concibe como rasgos de la biología oficial, una ciencia sustentada en procesos observacionales y lógicos. No obstante los aportes de la lógica y de la observación en la construcción y validación del conocimiento, queda claro que, para estos sujetos, ellos no bastan para dotar del status de absoluto al saber logrado, ya que éste será irremediablemente provisorio, dados los límites de la empiria y la razón.

Lo señalado dificulta la posibilidad de adscribirlos a algunas de las categorías habitualmente empleadas para caracterizar las creencias epistemológicas sobre la naturaleza de la ciencia. De hecho, autores como Petrucci y Dibar Ure (2001) advierten acerca de los riesgos que implica clasificar a los estudiantes o profesores en función de categorías epistemológicas relativas a las posiciones empirista, positivista, kuhniana, entre otras. Sostienen que en investigaciones de estas características es frecuente hallar referencias al inductivismo ingenuo, al empirismo y al positivismo como si se tratara de una misma postura. En lo concerniente a nuestros resultados, puede decirse que el contenido de las creencias de estudiantes y becarios parece mostrar atributos mixtos, donde se amalgaman rasgos del relativismo y del pragmatismo, y en menor medida del realismo (Vázquez, Acevedo, Manassero y Acevedo, 2001).

A continuación, se retoman las preguntas iniciales para organizar el desarrollo de las consideraciones finales.

\section{¿Cuál es el contenido de las creencias que sustentan estudiantes $y$ becarios sobre el carácter de la ciencia y las operaciones del quehacer científico?, ¿Cuáles son sus creencias respecto de las propiedades de las teorías y el lugar de la observación?}

Guando se trata de la naturaleza de la ciencia y sus propiedades definitorias, ambos grupos se inclinan hacia el sistema de reglas, quizás porque perciben que el emergente más notorio en este sentido está puesto en la normatividad propia del método, aspecto en torno al cual dicha ciencia ejerce un fuerte control mediante mecanismos institucionales de evaluación en diversas instancias de ese quehacer. Buena parte de sus justificaciones remarcan la dimensión normativa de la ciencia, apelando a cuestiones del método que es donde se sustancia dicha normatividad de forma muy elocuente.

$\mathrm{Al}$ definir las operaciones sustanciales del quehacer cientifico, el especular o cualquier otro tipo de actividad cognitiva vinculada con la generación de teoría no es denotada como propiedad decisiva, probablemente porque la formación disciplinar enfatiza en las actividades observacionales por sobre las generadoras de conocimiento. Así, teorizar es hacer ciencia, si bien esta acción no es suficiente per se, pues se requiere de una segunda instancia en la cual la observación cumple un papel trascendente. 
El reconocimiento de la ciencia como sistema de reglas construido y acordado convencionalmente para elaborar explicaciones útiles, sugiere la intersubjetividad como una propiedad puesta al servicio de acordar que será o no científico. Asimismo, cuando estudiantes y becarios privilegian la verificación y la observación como operaciones centrales de la dinámica científica, la ciencia pareciera encontrar allí el anclaje en lo real y, por ende, en el conocimiento objetivo.

Cuando se atribuyen propiedades a las teorías científicas, estudiantes ni becarios ponen en discusión su carácter conjetural, como tampoco su posibilidad de aproximación a la realidad, en tanto que sí es un aspecto discutido la relación de la teoría con la observación, pues se la piensa como derivada de ésta, o bien como confrontada con ella. La primera posición supone una realidad más objetiva, en tanto asume que desde la empiria se pueden construir verdaderos enunciados teóricos por inducción, a diferencia de la segunda que concede a la observación un papel meramente justificatorio. Igualmente, no hay acuerdo acerca de si la teoría es la mejor explicación, o una entre varias posibles. La primera alternativa acepta que ésta es la más congruente con el mundo objetivo, en tanto que la segunda no supone una referencia externa que admita mejores o peores aproximaciones a lo real.

En lo atinente al papel de la observación en el vínculo entre sujeto y objeto, la principal atribución se orienta en un sentido fundamentalmente justificatorio $y$ no heurístico. Estudiantes y becarios coinciden en que observar implica mediar entre la teoría y la realidad, mediación que, como lo advierte Bar (2013), no supone tornar lo posible e ideal en real, sino sólo representarlo en toda la acepción del término. Dicho de otro modo, volver a presentar en los hechos (lo objetivo) lo que antes estuvo en la mente (lo subjetivo).

Para unos y para otros, la observación es la estrategia sine qua non para aproximarse al objeto, aún con todas las debilidades que ésta supone, pues no puede rescatar per se lo que el objeto verdaderamente es, sino sólo una representación más o menos acabada del mismo. El hecho de que lo observado deba ser interpretado da muestras de que la estrategia es imperfecta, pues amerita leer el objeto desde algún lugar, dotarlo de sentido, otorgarle propiedades y adscribirlo a un sistema categorial.

La observación conlleva la idea de captación y manipulación del objeto, y en este sentido es objetiva, si bien dicha propiedad se torna relativa cuando se advierte que hay al menos dos factores que inciden fuertemente en sus resultados; el primero está dado por los límites de los sentidos, el segundo, por los conceptos y expectativas de los observadores. Los fuertes acuerdos en la biología, en tanto ciencia monoparadigmática, posibilitan ponerle un cerco a las operaciones sensoriales y a las teorías para que transiten por un solo camino donde la subjetividad pueda ser "controlada".

Desde lo epistemológico, puede decirse que, si bien no desconocen el papel del sujeto en la construcción del conocimiento, mantienen la creencia de que tal construcción logra efectivamente una aproximación al objeto, encarnada en el dato con sus propiedades de objetividad, ya se encuentre éste al inicio o al final de los procesos, mediante el sistema de normas que enmarcan al método. Las atribuciones referidas al carácter normativo de su ciencia, así como la función del método en el logro de la objetivación del fenómeno y la concreción del dato, son creencias construidas en el ámbito formativo que se sustanciaron conforme con un modelo asimilado a la concepción de ciencia normal kuhniana. En tal sentido, dicha "creencia normalizada" otorgará y asegurará cierta confiabilidad para el uso y desarrollo de los conceptos aceptados por la disciplina, y con ella el afianzamiento del paradigma imperante.

Lo antes dicho daría cuenta de una fuerte impronta de la formación inicial en la construcción de las creencias y significados desde los cuales estos sujetos connotan, no sólo la naturaleza de la ciencia y del quehacer científico, sino también su propia 
disciplina. En otros términos, los aprendizajes y experiencias arraigados en el contexto de educación caracterizado por Echeverría (1998) trazan, en buena medida, los horizontes de sentido en torno a los cuales se piensan la disciplina y sus praxis.

\section{¿Qué matices presentan las creencias examinadas entre quienes optaron por un trayecto de formación para la investigación?}

La reflexión en torno a la presencia de posibles matices en las creencias de los becarios respecto de las sustentadas por los estudiantes, amerita destacar marcadas semejanzas por sobre sutiles diferencias. $\mathrm{El}$ análisis pone de manifiesto un notable margen de acuerdo en la mayoría de los aspectos. Lo parcialmente distintivo para uno y otro grupo, se circunscribe al modo en que enunciaron y justificaron las concepciones acerca de la naturaleza de la ciencia. Asimismo, las pocas diferencias corresponden a la frecuencia de las elecciones para algunas de las alternativas ofrecidas en los diferentes planteamientos, aunque prevalecen las mismas orientaciones en sus respuestas.

Frente a estas consideraciones, surge un nuevo interrogante ¿cómo puede explicarse que las creencias de los jóvenes investigadores no muestren mayores modificaciones en relación con aquellas que sostienen los estudiantes? Una explicación posible es que los aspectos indagados respecto a la ciencia y a los procesos implicados en la producción de conocimiento constituyan elementos sustanciales de la organización conceptual de la disciplina. La formación inicial, y en buena parte, la formación de posgrado en biología, acude a procesos de normalización necesarios en pos de lograr sujetos que conozcan debidamente tanto las normas de actuación para vincularse con sus objetos, como el desarrollo de competencias que les permitan afirmar o negar algo en torno a ellos.

El contenido de las creencias examinadas podría remitir a cuestiones medulares en la constitución de las praxis disciplinares de los/as alumnos/as, por lo que las creencias conformadas por los becarios durante la formación de grado no serían susceptibles de modificación inmediata. Cabe destacar que los trayectos institucionales de formación para la investigación (la beca) son acotados en el tiempo, y posiblemente insuficientes para promover cambios sustanciales en las creencias epistemológicas de estos sujetos, constituidas en estrecha vinculación con el modelo de ciencia que se les ha enseñado.

Otra interpretación compete al carácter de estos trayectos formativos. Si bien es cierto que suponen una experiencia más protagónica por parte del becario en la producción de conocimiento disciplinar, su actividad se encuentra aún fuertemente normada por tratarse de investigadores noveles en el campo. Se trata de un quehacer que responde considerablemente a una lógica tutorial, en la cual los márgenes de autonomía continúan siendo estrechos (sus propios proyectos se inscriben en líneas de investigación ya instituidas, gran parte de las decisiones respecto de sus planes de trabajo son determinadas por sus directores, sus acciones están reguladas por plazos preestablecidos). Tales cuestiones van en detrimento de procesos autónomos, reflexivos y creativos, que permitan problematizar el modelo de ciencia en torno al cual se han formado para pensar otros posibles.

Es previsible que el logro de las metas en las que hace foco el desarrollo de los trayectos de beca, requiera de dispositivos que "aseguren" cierta eficacia en la concreción del proceso, eficacia que se traduce en la finalización del doctorado y en la obtención de las certificaciones pertinentes. Ésta se sustenta en un contexto resolutivo, expeditivo y con alta certidumbre, que toma cuerpo en la adopción de marcos referenciales y metodológicos consolidados y suficientemente debatidos en la disciplina.

Así, el proceso, aunque eficiente en términos del costo asumido y el beneficio logrado, da pocas condiciones para la construcción de saberes teóricos relevantes, o la inauguración de debates profundos 
al interior de la disciplina. Lo heurístico queda relegado a la ampliación de la base empírica, sin que ello ponga en riesgo el cimiento conceptual sobre el que se construyó la tesis. En estos términos, los modelos de la formación inicial heredados, sumamente prescriptivos y canónicos, todavía resultan eficaces

\section{Referencias}

Acevedo Díaz, J. (2000), "Algunas creencias sobre el conocimiento científico de los profesores de educación secundaria en formación inicial”, Bordón, vol. 52, núm.

1, pp. 5-16, <http://www.oei.es/salactsi/acevedol8. htm> [Consulta: octubre de 2006].

Aguirre, V. (2013), "Trayectorias de formación de los becarios de investigación en Ciencias Naturales en dos facultades de la Universidad Nacional del Nordeste: un estudio interpretativo", Tesis de Maestría, Argentina, Universidad Nacional del Nordeste.

Alexander, P. (1992), "Domain knowledge: evolving themes and emerging concerns", Educational Psychologists, núm. 27, pp. 33-51, DOI: http://dx.doi.org/10.1207/ s15326985ep2701_4 [Consulta: octubre de 2006].

Alvarado Rodríguez, María E. y F. Flores Camacho (2001), "Concepciones de ciencia de investigadores de la UNAM. Implicaciones para la enseñanza de la ciencia", Perfiles Educativos, vol. XXIII, núm. 92, pp. 32-53, <http://www.redalyc.org/pdf/132/13209203. pdf $>$ [Consulta: marzo de 2009].

Bar, A. (2013), "Saberes y prácticas cognitivas en el contexto de la formación disciplinar en Biología", Tesis doctoral, Argentina, Universidad Nacional del Nordeste.

Biglan, A. (1973), "Relationships between subject matter characteristics and the structure and output of university departments", Fournal of Applied Psychology, vol. 57, núm. 3, pp. 204-213, <http://edshare.soton. ac.uk/15017/2/Biglan_-_1973_-_Relationships_ between_subject_matter_characteristics_and_the_ en los inicios de la formación de posgrado, lo que no contribuye a poner en discusión las nociones en torno al quehacer científico, como del mismo modo, a instalar formas de pensamiento reflexivo y crítico, características distintivas del acervo científico.

structure_and_output_of_university_departments. pdf $>$ [Consulta: abril de 2009].

Buehl, M. y P. Alexander (2001), "Beliefs about academic knowledge", Educational Psychology Review, vol. 13, núm. 4, pp. 385-418, <https://link.springer.com/article/10. 1023/A:101 1917914756> [Consulta: marzo de 2009].

Buehl, M. y P. Alexander (2006), "Examining the dual nature of epistemological beliefs", International fournal of Educational Research, vol. 45, núm. 1-2, pp. 28-42, <https://eric.ed.gov/?id=EJ746403> [Consulta: marzo de 2009].

BriceñoMartínez, J.yA.Benarroch(2013), “Concepciones y creencias sobre ciencia, aprendizaje y enseñanza de profesores universitarios de ciencias", Revista Electrónica de Investigación en Educación en Ciencias, vol. 8, núm.1, pp. 24-41, <http://www.scielo.org.ar/pdf/reiec/v8n1/ v8nla03.pdf> [Consulta: noviembre de 2013].

Corral, N. y A. Bar (2012), "Construcción de racionalidades disciplinares", Cinta de Moebio, núm. 45, pp. 215234, <https://scielo.conicyt.cl/pdf/cmoebio/n45/ art03.pdf> [Consulta: enero de 2013].

De la Lama García, A., M. Castillo Mussot y M. De la Lama Zubirán (2013), “¿Existen diferencias en las creencias que regulan las investigaciones de los científicos naturales y sociales? 185 investigadores responden", Argumentos, núm. 71, pp. 39-66, <http:// www.scielo.org.mx/pdf/argu/v26n71/v26n71a3.pdf> [Consulta: febrero de 2014].

Echeverría, J. (1998), Filosofia de la ciencia, Madrid, Akal. Fabbri, P. (1999), El giro semiótico, Barcelona, Gedisa. 
García, M. B., M. Mateos Sanz y S. Vilanova (2011), "Contenido y naturaleza de las concepciones de profesores universitarios de biología sobre el conocimiento científico", Revista Electrónica de Enseñanza de las Ciencias, vol. 10, núm. 1, pp. 23-39, <http://reec.uvigo.es/volumenes/volumen10/ ART2_Vol10_N1.pdf> [Consulta: junio de 2012].

Giaccaglia, L. (2007), "La metáfora en la investigación", La Trama de la Comunicación, vol. 12, pp. 201-205, <http://www.redalyc.org/pdf/3239/323927062014. pdf $>$ [Consulta: junio de 2012].

Hofer, B. y P. Pintrich (1997), "The development of epistemological theories: beliefs about knowledge and knowing and their relation to learning", Review of Educational Research, vol.67, núm. 1,pp. 88-140, <http:// cedu.niu.edu/ $\sim$ walker/research/Epistemological $\% 20$ Theories.pdf> [Consulta: noviembre de 2011].

Hofer, B. (2006), "Domain specificity of personal epistemology: resolved questions, persistent issues, new models", International Fournal of Educational Research, vol. 45, núms. 1-2, pp. 85-95, DOI: http://dx.doi. org/10.1016/j.ijer.2006.08.006 [Consulta: abril de 2009].

Kövecses, Z. (2002), Metaphor. A practical introduction, Oxford/Nueva York, Oxford University Press.

Kuhn, T. (1971), Estructura de las revoluciones cientificas, México, FCE.

Lakoff, G. y M. Johnson (1980), Metaphors we live by, Chicago, University of Chicago Press.

Leal-Soto, F. (2010), “Creencias epistemológicas generales, académicas y disciplinares en relación con el contexto", Universitas Psychologica, vol. 9, núm. 2, pp. 381-392, <http://pepsic.bvsalud.org/pdf/up/v9n2/ v9n2a07.pdf> [Consulta: mayo de 2012].

Lipman, M. (1997), Pensamiento complejo y educación, Madrid, Ed. de la Torre.

Manassero Mass, M. y A. Vázquez Alonso (2000), "Creencias del profesorado sobre la naturaleza de la ciencia", Revista Interuniversitaria de Formación del Profesorado, núm. 37, pp. 187-208, <https://dialnet. unirioja.es/servlet/articulo? codigo $=118066>$ [Consulta: junio de 2006].
Marciales Vivas, G. (2003), "Pensamiento crítico: diferencias en estudiantes universitarios en el tipo de creencias, estrategias e inferencias en la lectura crítica de textos", Tesis Doctoral, Madrid, Facultad de Educación, Universidad Complutense de Madrid, <http://biblioteca.ucm.es/tesis/edu/ucm-t26704. pdf $>$ [Consulta: febrero de 2009].

Pérez Echeverría, M., M. Mateos, N. Scheuer y E. Martín Ortega (2006), "Enfoques en el estudio de las concepciones sobre el aprendizaje y la enseñanza", en Nuevas formas de pensar la enseñanza y el aprendizaje. Las concepciones de profesores y alumnos, Barcelona, Graó, pp. 55-94.

Petrucci, D. y M. C. Dibar Ure (2001), "Imagen de la ciencia en alumnos universitarios", Enseñanza de las Ciencias, vol. 19, núm. 2, pp. 217-229, <http:// redined.mecd.gob.es/xmlui/handle/11162/23548> [Consulta: mayo de 2007].

Rabanal Moreno, E. y M. Quintanilla Gatica (2010), "Caracterización de las concepciones epistemológicas del profesorado de Biología en ejercicio sobre la naturaleza de la ciencia”, Revista Electrónica de Enseñanza de las Ciencias, vol. 9, núm. 1, pp. 11-124, <https:// dialnet.unirioja.es/servlet/articulo?codigo $=5045901>$ [Consulta: agosto de 2011].

Samaja, J. (1993), Epistemología y metodología. Elementos de una teoría para la investigación científica, Buenos Aires, EUDEBA.

Santibáñez, C. (2009), "Metáforas y argumentación: lugar y función de las metáforas conceptuales en la actividad argumentativa”, Signos, núm. 70, pp. 245-269, <https://dialnet.unirioja.es/servlet/ articulo?codigo=3030873> [Consulta: febrero de 2011].

Thomaz, M., M. Cruz, I. Martins y A. Cachapuz (1996), "Concepciones de futuros profesores del primer ciclo de primaria sobre la naturaleza de la ciencia: contribuciones de la formación inicial", Enseñanza de las Ciencias, vol. 14, núm. 3, pp. 315-322, <https:// ddd.uab.cat/record/22316> [Consulta: noviembre de 2010].

Vázquez Alonso, A., M. Manassero Mas y M. Talavera (2010), "Actitudes y creencias sobre naturaleza de la 
ciencia y la tecnología en una muestra representativa de jóvenes estudiantes", Revista Electrónica de Enseñanza de las Ciencias, vol. 9, núm. 2, pp. 333-352, <http:// reec.uvigo.es/volumenes/volumen9/ART3_Vol9_ N2.pdf> [Consulta: febrero de 2012].
Vázquez, A., J. Acevedo, M. Manassero y P. Acevedo (2001), "Cuatro paradigmas básicos sobre la naturaleza de la ciencia", Argumentos de razón técnica, núm. 4, pp. 135-176, <https://idus.us.es/xmlui/ handle/11441/21704> [Consulta: febrero de 2014].

\section{Cómo citar este artículo:}

Silvestri, Lisel-Irina, Miriam-Liset Flores y Aníbal-Roque Bar (2020), “La perspectiva de estudiantes y jóvenes investigadores en biología acerca de la ciencia", Revista Iberoamericana de Educación Superior (RIES), vol. XI, núm. 32, pp. 87-107, DOI: https://doi.org/10.22201/ iisue.20072872e.2020.32.810 [Consulta: fecha de última consulta]. 